\title{
Constraining the magnetic field in the X-ray corona of Cygnus X-1 with INTEGRAL*
}

\author{
Melania Del Santo \\ INAF/IAPS, Rome, Italy \\ E-mail: melania.delsanto@iaps.inaf.it \\ Julien Malzac, Renaud Belmont, Laurent Bouchet \\ CNRS, IRAP, Toulouse, France
}

\section{Giovanni De Cesare}

IAPS, Rome, Italy

\begin{abstract}
We present six years of INTEGRAL (IBIS, SPI and JEM-X) observations of the BH binary Cygnus $\mathrm{X}-1$. We produced 12 high-quality broad-band spectra (3 keV-1 MeV), representative of the whole range of spectral shapes observed in this source. We have studied the evolution of the physical parameters of the accretion flow across spectral transitions, by using two hybrid thermal/nonthermal Comptonization models. In particular, we used for the first time on Cyg X-1 data, the new model BELM which gives constraints on the magnetic field in the X-ray corona. We find that in the softer states, the magnetic field is at most of the order of $1 E+06 \mathrm{G}$. In the harder states, if the non-thermal excess observed above a few hundred $\mathrm{keV}$ is produced in the same region as the bulk of the thermal Comptonization, the upper limit on the magnetic field is about $1 \mathrm{E}+05 \mathrm{G}$. On the other hand, this high-energy excess may be produced in the jet (as recently suggested by INTEGRAL polarization measurements): in this case the constraints on the magnetic field in the hard states are somewhat relaxed and the upper limit rises to $1 \mathrm{E}+07 \mathrm{G}$.
\end{abstract}

"An INTEGRAL view of the high-energy sky (the first 10 years)" 9th INTEGRAL Workshop and celebration of the 10th anniversary of the launch,

October 15-19, 2012

Bibliotheque Nationale de France, Paris, France

* Based on observations with INTEGRAL, an ESA project with instruments and science data centre funded by ESA member states (especially the PI countries: Denmark, France, Germany, Italy, Switzerland, Spain), Czech Republic and Poland, and with participation of Russia and the USA.

†peaker. 


\section{Introduction}

In the simplest classification, Black Hole binaries (BHBs), and in particular Cyg X-1, are observed in two main spectral states [24]: the hard state (HS) with a X/ $\gamma$ spectrum believed to originate from thermal Comptonization in a hot electrons dominated cloud (the so-called "corona"; Sunyaev \& Titarchuk 1980); the soft state (SS) showing a prominent $\sim 1 \mathrm{keV}$ black-body associated with the optically thick accretion disc [20] plus a power-law tail, usually explained as Comptonization by a non-thermal electrons distribution[25, 9].

Although the scattering electrons have a predominantly Maxwellian energy distribution in the HS, there are indications that the electron distribution may have some high-energy tail i.e., it is hybrid, thermal/non-thermal. The first evidence has been provided by CGRO/COMPTEL observations at $\mathrm{E}>500 \mathrm{keV}$ of Cyg X-1 in hard state [13]. Then, with the INTEGRAL mission, this feature has been observed many times in a number of BHBs and BH candidates (see [5], i.e. 1E 1740.7-2942 [2], GX 339-4 [6], Cyg X-1 [3, 10]. Alternatively, basing on the strong polarization factor measured in the Cyg X-1 emission above $400 \mathrm{keV}[12,11]$, it has been proposed that the high-energy excess in hard state is likely to be produced in jet through synchrotron emission in a very coherent magnetic field.

The different electron distributions in HS and SS could be caused by kinetic processes and changing because of varying physical conditions in the corona $[15,19]$. These authors developed two independent codes and showed that in presence of a magnetic field in the corona, the electron distribution can appear thermal, even when acceleration mechanisms would produce non-thermal distributions. They have presented a rough 'fit by eye' of the average CGRO data of Cyg X-1 using these models, while [7] performed a statistical fit of INTEGRAL spectra by using the same model of [1].

\section{Cyg X-1 accretion flow study with INTEGRAL observations}

We have analyzed 6 years (2 Ms) of INTEGRAL public data and obtained 12 broad-band (3 $\mathrm{keV}-1 \mathrm{MeV}$ ) spectra. We have studied the whole range of observed spectral shapes in Cyg X1 by fitting them with two different Comptonization models, namely EQPAIR [4] and BELM [1]. This allowed us to constrain the physical conditions in the corona and disc and determine how the physical parameters change during the spectral evolution. In particular, fits with BELM set quantitative constraints on the strength of the magnetic field in the corona of Cyg X-1 for the first time.

The differences between EQPAIR and BELM can be briefly summarized as follow: in the first model, there is no magnetic field in the corona considered; the Comptonizing electrons population is hybrid thermal (Maxwellian) and non-thermal $\left(\Gamma_{\text {inj }}\right)$; seed photons come from the thin accretion disc. In the second model, the magnetic field $\mathrm{B}$ is taken into account and expressed by the magnetic compactness $1_{B} \propto \mathrm{B}^{2}$; there is only one population of non-thermal electrons injected with $\Gamma_{\text {inj }}$ and then thermalized via Synchrotron boiler [8]; the seed photons are due to both synchrotron radiation and disc photons.

\subsection{Results with both unmagnetized and magnetized models}

Details on the models, spectral analysis variability study and results are reported in [7]; here, we only summarize and discuss few results. We only point out that for the first study we used 

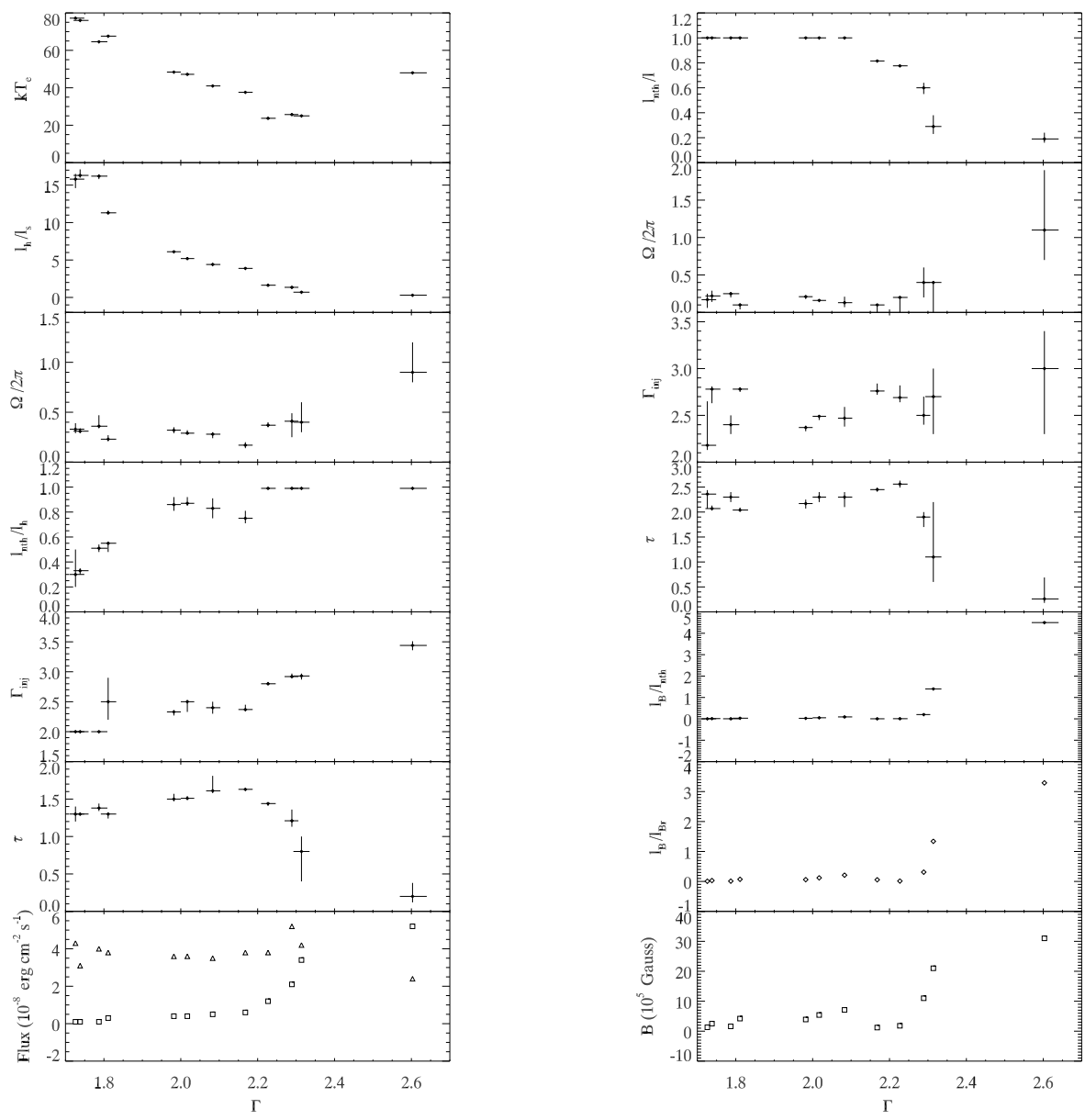

Figure 1: EQPAIR (Left) and BELM (Right) spectral parameters reported in [7] (Tab. 3 and 4, respectively) vs the $\Gamma$ slope of the IBIS/ISGRI spectra fit with a simple power law in the range $30-100 \mathrm{keV}$.

BELM assuming a Synchrotron Self Compton (SSC) emission and pure non-thermal acceleration; we did not include any thermal or Coulomb heating.

The parameters $\tau_{\mathrm{p}}, k T_{\mathrm{in}}, l_{\mathrm{nth}}\left(\right.$ or $\left.l_{\mathrm{h}} / l_{\mathrm{s}}\right)$ and the reflection amplitude have a similar behaviour when using the two models (Fig. 1). The fact that $l_{\text {nth }} / l$ (with BELM) is lower in softer state (steep $\Gamma$ ) is equivalent to the trend observed for the ratio $l_{\mathrm{h}} / l_{\mathrm{S}}$ in EQPAIR. We note that the electron acceleration index $\Gamma_{\text {inj }}$ is better constrained by the data in the magnetized model, since it also affects the number of synchrotron emitting electrons which in turns control the temperature of the Maxwellian electrons and the slope of the X-ray spectrum.

A common interesting trend (shown in Fig. 1) is that the Thomson optical depth is lower in softest states $\left(\tau_{\mathrm{p}} \simeq 0.2\right.$ in SS). It increases significantly from SS to intermediate states and is approximately constant in the hard states. This change by a factor of roughly 10 could be associated either to a change in electron density or in the size of the corona (since $\tau \propto n_{e} R$ ). If this is the size of the corona that changes then this would imply that the corona is larger in the HS than in the SS by the same factor. This would be in agreement with the decrease of the inner radius of the accretion disc and faster rapid variability in the SS which both suggest a smaller emitting region 


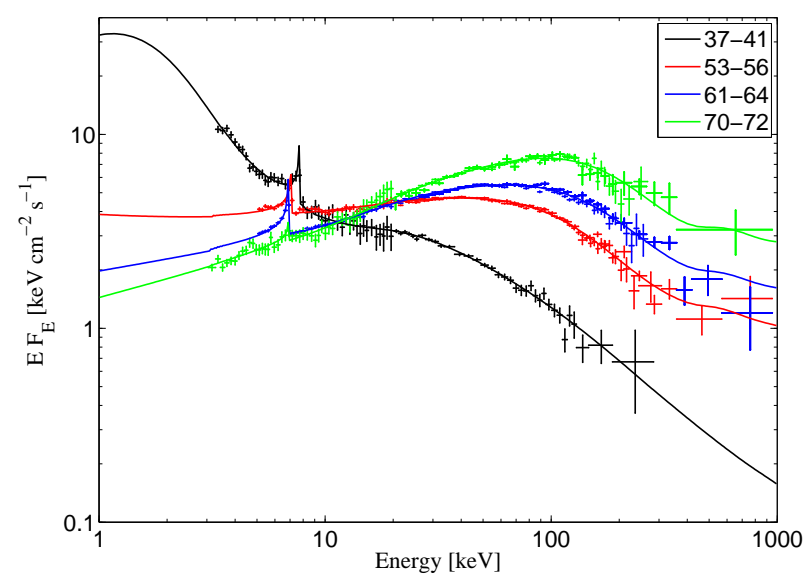

Figure 2: Joint JEM-X, IBIS and SPI energy spectra of Cyg X-1 during four different spectral states fitted with the BELM model with pure non-thermal acceleration plus DISKLINE and REFLECT (see [7]).

in the SS. But the electron density could also drive the change in Thomson depth. This could be the case if the material in the corona gradually condensates into the accretion disc when the source evolves toward softer states. Moreover, we note that values of $\tau$ higher than 1 in the HS does not reproduce ADAF models [16]. Latest elaboration of ADAF models reproduce high $\tau$, even though they require high values of $B$ [17]

Non-thermal acceleration appears to be required to fit all spectra with EQPAIR. This indicates that a non-thermal electrons population may also be present in the corona (or elsewhere), together with the thermal population even during the hard states. This is in agreement with previous studies performed on intermediate and hard states of BH binaries, as also reported in Sec. 1.

We observe dramatic variation of the thermal disc flux (of a factor of 50), low $\mathrm{kT}_{e}$ in $\mathrm{SS}$ and Comptonized component almost constant (a factor of 2; Fig. 1, left): we then conclude that spectral transition are driven by the soft cooling.

From our fits with BELM, it is clear that in the soft state the accretion disc photons represent the main source of soft seed photons (see Fig. 3). However, with BELM we observe that in the HS the synchrotron photons may dominate. [21] have argued that in a number of BHCs synchrotron seeds should dominates at luminosities below about a percent of the Eddington limit, while disc seed photons should dominate at higher luminosities. It is interesting to note that Cyg X-1 has a stable luminosity located around this transition. It is therefore likely that both the accretion disc and the magnetic field contribute to seed the Comptonization process.

Concerning the magnetic field intensity, we found that in the hard state the upper limit on the magnetic field is $10^{5} \mathrm{G}$. It is then interesting to express the magnetic field compactness as a fraction of the equipartition compactness [7] and estimate the ratio $1_{B} / 1_{B r}$ which is independent of the uncertainties on the source size and distance. In the harder states we found strongly subequipartition magnetic fields $\left(1_{B} / 1_{B r} \ll 1\right)$ which implies that $\mathrm{B}$ cannot power the corona. On the other hand, in the soft states $B$ is super-equipartition $\left(1_{B} / l_{B r}>1\right)$ and of the order of $10^{6} \mathrm{G}$ which is consistent with the corona powered by the magnetic energy. These results are in agreement with qualitative results of [19] (with a different code) and [15], as well as consistent with analytic 

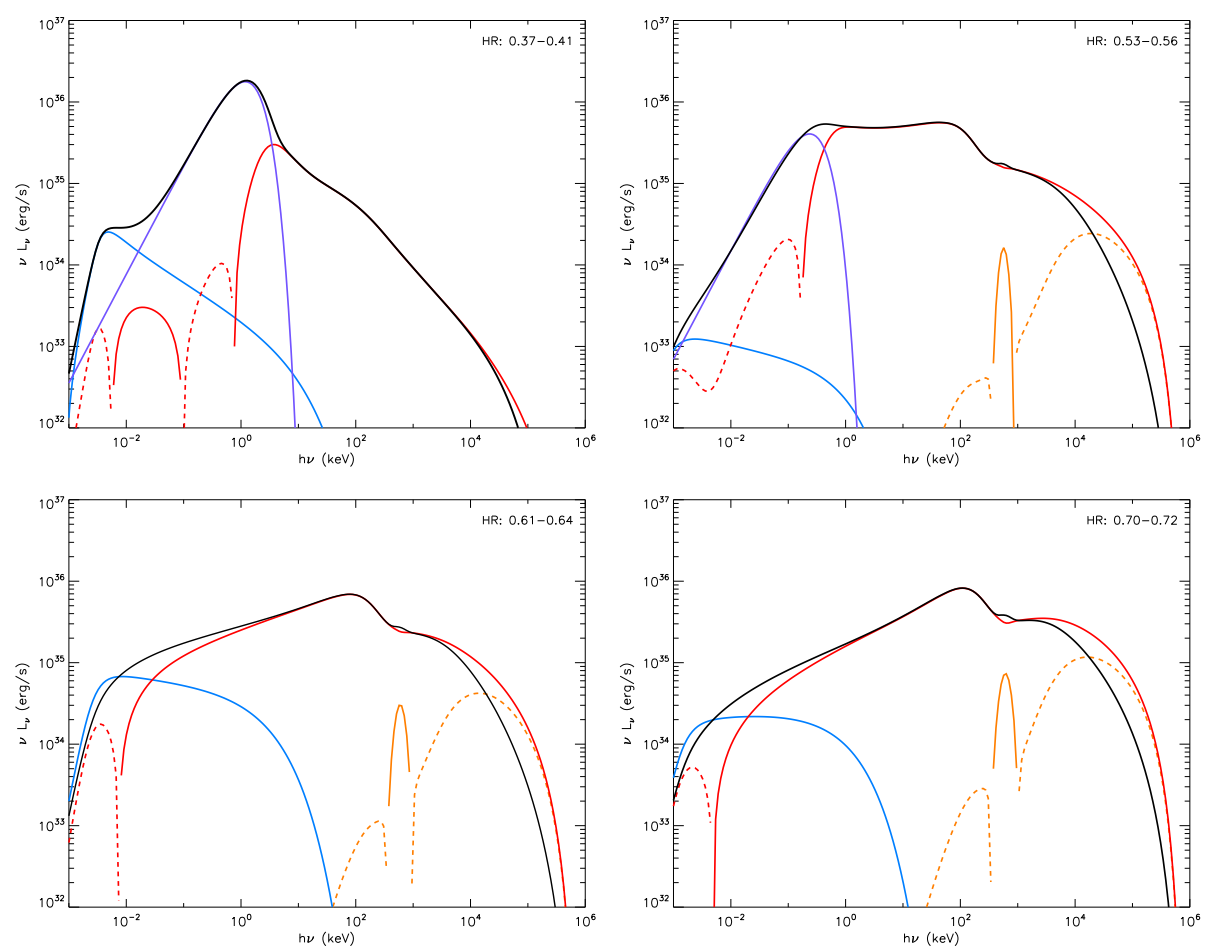

Figure 3: BELM total models (black) corresponding to fits of the four spectra shown in Fig. 2 and the components: Compton (red), pair annihilation/production (orange), synchrotron (blue), soft black body photons (purple). Solid lines correspond to positive contribution to the spectrum, dashed lines correspond to negative contribution.

estimation of [23]. We can conclude that our result are not model dependent.

\subsection{High energy emission from different regions than the corona?}

We have also studied the case that the tail observed in the hard states is not produced in the same region as the bulk of the thermal Comptonization, but somewhere else (jet? [12, 26] another corona region? [16]). Then, the hard spectra below the cutoff could also be reproduced by SSC models with pure thermal heating. In this case the exact heating mechanism is of little importance for spectral modeling, because whatever the heating mechanism the resulting electron distribution is very close to a pure Maxwellian. In BELM this electron thermal heating mechanism is assumed to be Coulomb collisions with a distribution of hot thermal protons [15]. Then, the constraints on the magnetic field are expected to be different.

As expected, the broadband spectrum can not be fitted with this pure thermal model, since it does not reproduce the highest energy data (above $200 \mathrm{keV}$ ), which either requires a significant level of non-thermal acceleration or must originate in a different region. When excluding data above $200 \mathrm{keV}$, we obtained good fits (see Tab. 5 in [7]).

Thus, by assuming that the highest energy emission originates from a different region, we obtain that the magnetic field in the hard states is stronger by a factor of 100 compared to that estimated in the SSC model. The magnetic field is now allowed to be strongly super equipartition $\left(1_{B} / 1_{B r} \gg 1\right)$ with typical upper limits of the order of $10^{7} \mathrm{G}$. In this case B is consistent with the 
magnetically dominated hot accretion flow model of $[17,18]$ which also produces the rather large Thomson depths observed.

\section{Acknowledgments}

MDS and GDC acknowledge financial support from the agreement ASI-INAF I/009/10/0 and from PRIN-INAF 2009 (PI: L. Sidoli). MDS and JM acknowledge financial support from Université Paul Sabatier. JM \& RB acknowledge support from PNHE in France. MDS thanks IRAP/CNRS (France) for hospitality. JM thanks the Institute of Astronomy of Cambridge (UK) for hospitality.

\section{References}

[1] Belmont R., Malzac J., Marcowith A., 2008, A\&A, 491, 617

[2] Bouchet L., et al., 2009, ApJ, 693, 1871

[3] Cadolle Bel M., et al, 2006, A\&A, 446, 591

[4] Coppi P. S., 1999, ASP Conf. Ser. 161: High Energy Processes in Accreting Black Holes, 161, 375

[5] Del Santo M., 2012, JPhCS, 354, 012003

[6] Del Santo M., et al., 2008, MNRAS, 390, 227

[7] Del Santo M., et al., 2013, MNRAS, in press (astro-ph/1212.2040)

[8] Ghisellini G., Guilbert P. W., Svensson R., 1988, ApJ, 334, L5

[9] Gierliński M., et al., 1999, MNRAS, 309, 496

[10] Jourdain E., Roques J. P., Malzac J., 2012a, ApJ, 744, 64

[11] Jourdain E., et al., 2012b, ApJ, 761, 27

[12] Laurent P.,et al., 2011, Science, 332, L438

[13] McConnell M. L., et al., 1994, ApJ, 424, 933

[14] Malzac J., et al., 2006, A\&A, 448, 1125

[15] Malzac J. \& Belmont R., 2009, MNRAS, 392, 570

[16] Malzac J., 2012, IJMPS, 8, 73

[17] Oda H., et al., 2012, PASJ, 64, 15

[18] Oda H., et al., 2010, ApJ, 712, 639

[19] Poutanen J., \& Vurm I., 2009, ApJL, 690, 97

[20] Shakura N. I., Syunyaev R. A., 1973, A\&A, 24, 337

[21] Sobolewska M. A., et al., 2011, MNRAS, 417, 280

[22] Sunyaev R. A., Titarchuk L. G., 1980, A\&A, 86, 121

[23] Wardziński, G., Zdziarski A. A., 2001, MNRAS, 325, 963

[24] Zdziarski A. A., et al., 2002, ApJ, 578, 357

[25] Zdziarski A. A., Gierkiński M., 2004, Progress of theoretical Physics, 155, 99

[26] Zdziarski A. A., Lubiński P., Sikora M., 2012, MNRAS, 423, 663 